\title{
Cotrafficking of SV2 and Synaptotagmin at the Synapse
}

\author{
Jia Yao, Amy Nowack, Patricia Kensel-Hammes, Richard G. Gardner, and Sandra M. Bajjalieh \\ Department of Pharmacology, University of Washington, Seattle, Washington 98195
}

Synaptic vesicles are specialized cycling endosomes that contain a unique constellation of membrane proteins. Proteins are sorted to vesicles by short amino acid sequences that serve as binding sites for clathrin adaptor proteins. Here we show that a tyrosine-based endocytosis motif in the vesicle protein SV2 is required for trafficking to synaptic vesicles of both SV2 and the calcium sensor protein synaptotagmin. Aberrant neurotransmission in cultured hippocampal neurons lacking SV2 was rescued by expression of wild-type SV2A, but not by SV2A-Y46A, a mutant containing a disrupted endocytosis motif in SV2A's cytoplasmic $\mathrm{N}$ terminus. Neurons expressing SV2A-Y46A had significantly more SV2 on the plasma membrane, indicating reduced internalization. A screen for proteins that preferentially bound wild-type SV2A identified multiple endocytosis-related proteins, and in vitro binding studies confirmed binding to the clathrin adaptors AP2, EPS15, and amphiphysin 2/Bin1. Neurons lacking SV2 contained less synaptotagmin and had a higher proportion of synaptotagmin on the plasma membrane. Expression of either wild-type SV2A or SV2A-Y46A restored synaptotagmin expression levels; however, only wild-type SV2A restored a normal proportion of synaptotagmin on the plasma membrane. These findings indicate that SV2 influences the expression and trafficking of synaptotagmin via separate mechanisms. Synaptic vesicles immunoisolated from SV2A/B double knock-out mice had significantly less synaptotagmin than vesicles isolated from wild-type mice. Our results indicate that SV2 plays a major role in regulating the amount of synaptotagmin in synaptic vesicles and provide an explanation for the observation that synapses lacking SV2 have fewer vesicles competent for calcium-induced fusion.

\section{Introduction}

Proteins unique to regulated secretion mediate the refinement of exocytosis that occurs at neuronal synapses. Among these is the membrane protein SV2, which is specific to secretory vesicles in neurons and endocrine cells. Mammals have three SV2 genes that encode isoforms termed SV2A, SV2B, and SV2C. SV2 is an essential protein. Mice lacking the most widely expressed isoform, SV2A, develop severe seizures and die within 3 weeks of birth (Crowder et al., 1999; Janz et al., 1999).

SV2A is the binding site of levetiracetam (Lynch et al., 2004), an Food and Drug Administration-approved drug that is currently used in the treatment of epilepsy (for review, see De Smedt et al., 2007), and which also shows promise for the treatment of anxiety disorders (Zhang et al., 2005; Kinrys et al., 2006, 2007), pain (Price, 2004; Dunteman, 2005; Enggaard et al., 2006), dyskinesias (McGavin et al., 2003; Bushara et al., 2005; Striano et al., 2007; Woods et al., 2008; Zivkovic et al., 2008), and posttraumatic stress disorder (Kinrys et al., 2006). At present, SV2 is the only drug target in synaptic vesicles.

Analyses of neurotransmission in mice lacking SV2A reveal a significant decrease in evoked vesicle fusion in both excitatory (Custer et al., 2006) and inhibitory (Crowder et al., 1999; Chang

Received Sept. 25, 2009; revised Jan. 20, 2010; accepted Feb. 7, 2010

This work was supported by National Institutes of Mental Health Grant R01 MH 059842 to S.B. We thank Drs. Beverly Wendland and John Scott for comments on this manuscript, Greg Martin of the University of Washington Keck Imaging Center for help with imaging, and Lisa Baldwin for animal husbandry and editorial comments on this manuscript.

Correspondence should be addressed to Sandra M. Bajjalieh, 1959 NE Pacific, Box 357750, University of Washington, Seattle, WA 98195. E-mail: bajjalie@uw.edu.

D01:10.1523/JNEUROSCI.4781-09.2010

Copyright $\odot 2010$ the authors $\quad 0270-6474 / 10 / 305569-10 \$ 15.00 / 0$ and Sudhof, 2009) CNS neurons, as well as in cultured chromaffin cells (Xu and Bajjalieh, 2001). This effect is limited to calciuminduced neurotransmission, as there is no difference in the frequency of spontaneous transmitter release (Crowder et al., 1999; Custer et al., 2006).

All SV2 isoforms bind the synaptic vesicle protein synaptotagmin (Schivell et al., 1996, 2005; Lazzell et al., 2004). Loss of SV2B, the predominant isoform in retinal photoreceptors, results in decreased levels of synaptotagmin 1 in photoreceptor synapses (Lazzell et al., 2004; Morgans et al., 2009). This suggests that SV2 influences the expression, trafficking, or stability of synaptotagmin. SV2 does not appear to affect vesicle formation, however, since synaptic morphology and vesicle density are not altered in SV2 knock-outs (Crowder et al., 1999; Janz et al., 1999; Morgans et al., 2009). Together, these observations suggest that SV2 could influence neurotransmitter release by regulating the amount of synaptotagmin in secretory vesicles. Here we report that mutation of a tyrosine-based endocytosis motif in SV2A results in a nonfunctional protein that disrupts the endocytosis of both SV2 and synaptotagmin. Thus, SV2 plays a crucial role in the trafficking of synaptotagmin to synaptic vesicles and regulates the effectiveness of calcium in inducing vesicle fusion by regulating the vesicle content of a major calcium sensor protein.

\section{Materials and Methods}

Plasmids. Site-directed mutagenesis of rat SV2A cDNA in pCDNA3.1 was performed using a QuikChange Site-Directed Mutagenesis kit (Stratagene). Tyrosines 46 and 443 were replaced with alanine. The primers used included the following: Y46Af, 5'-CGA AGG TCC GCC TCC CGC TTT GAG GAG GAG GAG-3'; Y46Ar, 5'-AAA GCG GGA GGC GGA CCT TCG GGA ATA TTC ATC CTG-3'; Y443Af, 5'-AGT CCA GAG GCC CGG CGC ATC ACT CTG ATG ATG-3'; and Y443Ar, 5'-GAT 
GCG CCG GGC CTC TGG ACT GAA GCA GGA GAG-3' (f and r denote forward and reverse primers, and italic letters indicate mismatches). To make the Y46A/Y443A double mutant, a NheI/SacII fragment in SV2AY443A pCDNA3.1 was replaced with the corresponding fragment from a SV2A-Y46A pCDNA3.1 construct. All constructs were sequenced to ensure that no additional mutations were introduced during PCR amplification. cDNAs encoding the mutant SV2As were subcloned in frame with enhanced green fluorescent protein (EGFP) in the vector pRRL. This produced vectors encoding SV2A-EGFP fusion proteins.

Primary hippocampal neuron culture and viral gene delivery. Microisland cultures of primary hippocampal neurons were prepared as described previously (Custer et al., 2006). Cultures were infected with lentivirus virions as described below and used for electrophysiological recording or $\mathrm{N}$-(3-triethylammoniumpropyl)-4-(6-(4-(diethylamino) phenyl)hexatrienyl) pyridinium dibromide (FM 4-64) labeling experiments at $12-16 \mathrm{~d}$ in vitro (DIV). Conventional hippocampal neuron cultures were prepared on six-well plates precoated with poly-D-lysine. Generally, $2-3 \times 10^{5}$ cells/well were maintained in astrocyte-conditioned neuron media supplemented with $80 \mu \mathrm{M}$ mitosis inhibitor (5fluoro-2'-deoxy-uridine; Sigma) to suppress the growth of astrocytes. Cultures were infected with lentivirus at 2-3 DIV and used for surface biotinylation experiments at 13-15 DIV.

Lentiviral gene delivery. Virions generated from lentivirus carrying the SV2A-EGFP cDNAs were produced in human embryonic kidney 293T (HEK293T) fibroblasts. Briefly, cells were cotransfected with packing plasmids $\mathrm{pLP} 1, \mathrm{pLP} 2$, and $\mathrm{pLP}$ VSV-G, and the carrier vectors containing SV2A-EGFP using calcium phosphate. Sixteen hours after transfection, the medium was replaced with fresh medium. Media containing virions were harvested twice from the culture. Media were filtered through 0.22 $\mu \mathrm{m}$ pore PVDF membranes and concentrated by centrifuging at $6700 \times$ $g$ for a minimum of $16 \mathrm{~h}$ at $4^{\circ} \mathrm{C}$. The virion pellets were resuspended in medium and stored at $-80^{\circ} \mathrm{C}$.

Immunocytochemistry. Antibody labeling of hippocampal neurons expressing exogenous SV2 was performed at 12-15 DIV as described previously (Custer et al., 2006) using an anti-synaptophysin monoclonal (1:2000 dilution; Millipore Bioscience Research Reagents) and a polyclonal antibody directed against SV2A (Bajjalieh et al., 1994) (1:1000 dilution). Immunolabeling of neurons cultured from SV2A/B double knock-out (DKO) mice used a polyclonal antibody against synaptotagmin 1 and a monoclonal antibody against synaptophysin. Antibody labeling was detected with fluorescent secondary antibodies (goat-antirabbit Alexa-Fluor 488 and goat-anti-mouse Alexa-Fluor 568, or goatanti-rabbit Alexa-Fluor 568 and goat-anti-mouse Alexa-Fluor 647; Invitrogen). Images were obtained with a confocal laser-scanning microscope (SP1 confocal; Leica) with a $100 \times$ oil-immersion objective (numerical aperture, 1.40). Images of SV2-expressing neurons were processed and assembled using Adobe Photoshop (Adobe Systems). Analysis of synaptotagmin 1 immunofluorescence was performed with MetaMorph image software with anti-synaptophysin labeling used to define synaptic puncta.

FM 4-64FX labeling. Coverslips on which DIV 11-15 mouse hippocampal neurons were cultured as for physiological analyses were rinsed in external solution (Custer et al., 2006) containing $2 \mathrm{~mm} \mathrm{CaCl}$ and $4 \mathrm{mM} \mathrm{MgCl}_{2}$, and challenged with a high-potassium solution [containing the following (in mM): $60 \mathrm{KCl}, 64 \mathrm{NaCl}, 30$ glucose, 20 HEPES, 2 $\mathrm{CaCl}_{2}, 4 \mathrm{MgCl}_{2}$, and 0.01 glycine] in the presence of $10 \mu \mathrm{g} / \mathrm{ml} \mathrm{FM} \mathrm{4-64FX}$ (Invitrogen) for $60 \mathrm{~s}$. Excess dye was removed with seven 1 min rinses in $\mathrm{Ca}^{2+}$-free external solution, two of which contained $300 \mu \mathrm{M}$ Advasep-7 (Biotium). Immediately after rinses, neurons were fixed in $4 \%$ paraformaldehyde, rinsed with $0.1 \mathrm{~m}$ glycine in PBS and mounted on glass slides for confocal microscopy. Images were taken using a Leica SP1 confocal microscope with equal exposure settings in the red (FM 4-64) channel and analyzed with MetaMorph image software. Images were thresholded based on SV2A-EGFP fluorescence to define synaptic locations. Regions of interest larger than four pixels were mapped onto the FM 4-64 signal, and the average intensity (total integrated intensity/area) of spots was determined. The average of these values in each image was calculated. The mean of wild-type images was used to normalize the data in that experiment. Five independent experiments were conducted. a
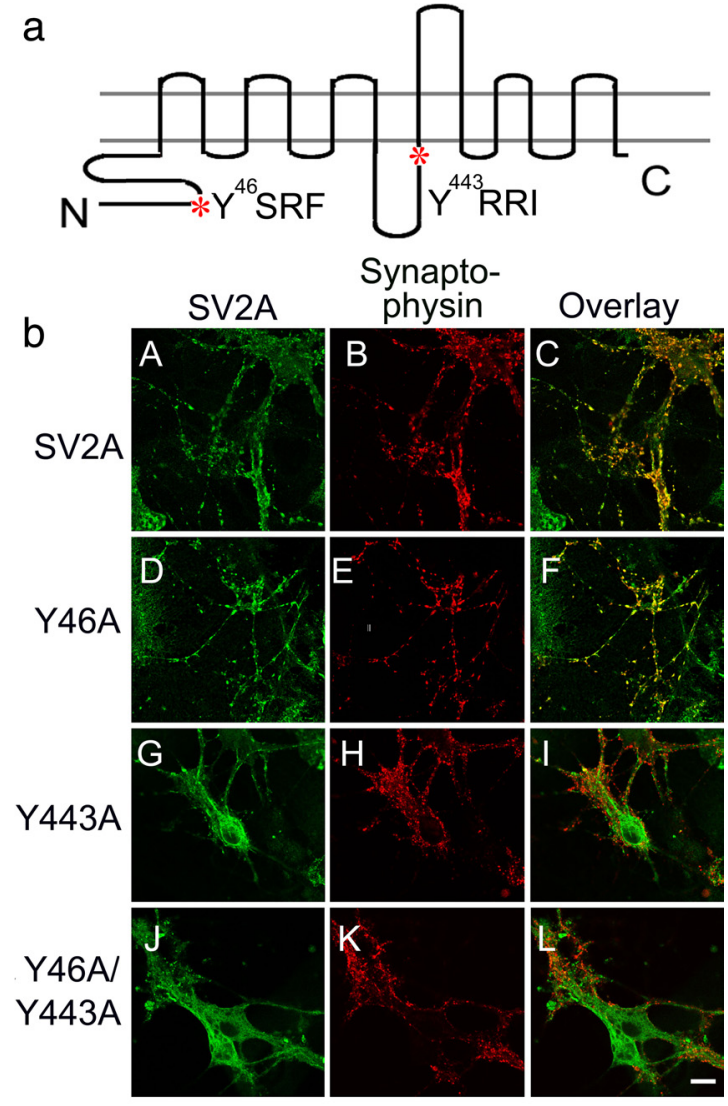

C
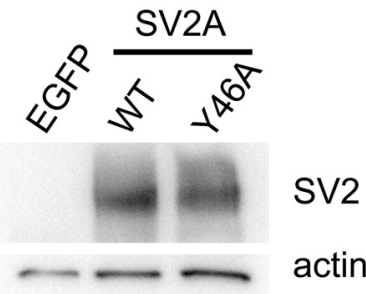

Figure 1. Expression of SV2A-EGFP fusion proteins in hippocampal neurons from SV2A/B DK0 mice. $\boldsymbol{a}$, Schematic of SV2A showing, with red asterisks, the location of two tyrosine-based endocytosis motifs. $N$ and $C$ denote the $N$ and $C$ termini, respectively. $\boldsymbol{b}$, SV2A and SV2A-Y46A colocalize with synaptophysin. Hippocampal neurons from SV2A/B DKO mice were cultured on microislands and infected with lentivirus encoding the indicated SV2A-EGFP protein. Neurons were labeled with anti-SV2A polyclonal and anti-synaptophysin monoclonal antibodies. Antibody binding was visualized with goat-anti-rabbit Alexa-Fluor 488 (green) and goat-antimouse Alexa-Fluor 568 (red). Shown are images collected with a confocal microscope. SV2AEGFP and SV2A-Y46A-EGFP produced punctate labeling $(\boldsymbol{A}, \boldsymbol{D})$ that colocalized with synaptophysin labeling $(\boldsymbol{B}, \boldsymbol{E})$. The yellow signal in $\boldsymbol{C}$ and $\boldsymbol{F}$ indicates colocalization of the SV2A and synaptophysin labeling. In contrast, SV2A-Y443A (G) and SV2A-Y46A/Y443A $(J)$ were diffusively distributed in the neurons and did not colocalize with synaptophysin $(\boldsymbol{H}, \boldsymbol{I}, \boldsymbol{K}$, and $\boldsymbol{L})$. Scale bar, $10 \mu \mathrm{m}$. C, SV2A and SV2A-Y46A are expressed at comparable levels. Shown is a representative Western blot analysis of conventional cultures expressing the indicated SV2A construct or EGFP. Blots were probed for SV2A and actin (as a loading control). SV2 net intensity was normalized to the net intensity of actin. On average, SV2A-Y46A was expressed at $\sim 80 \%$ the levels of wild-type SV2A.

Electrophysiological recording and analysis. Acquisition and analysis of whole-cell voltage-clamp recordings of synaptic responses in autaptic hippocampal neurons were performed as described previously (Custer et al., 2006), with the exception that recordings were performed under a controlled temperature between 21.0 and $22.5^{\circ} \mathrm{C}$.

Pull-down assays. All animal protocols used in these experiments were approved by the Institutional Animal Care and Use Committee of the University of Washington. Ten- to 12-week-old mice were killed by cervical dislocation. Brains were removed immediately and homogenized in 
a

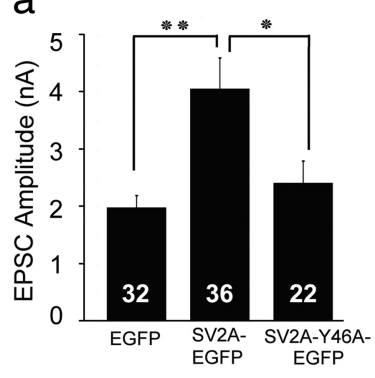

b

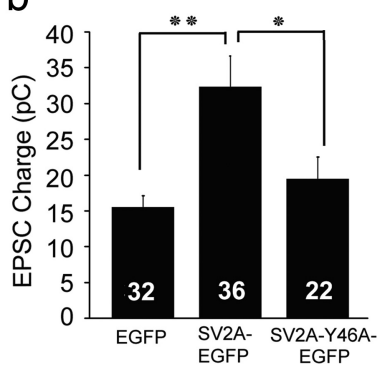

C

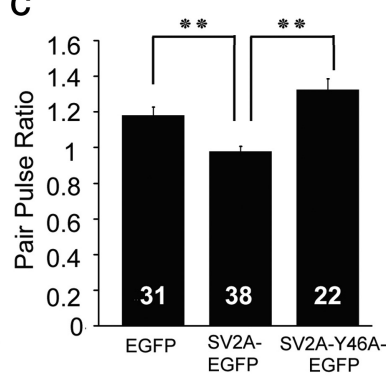

d
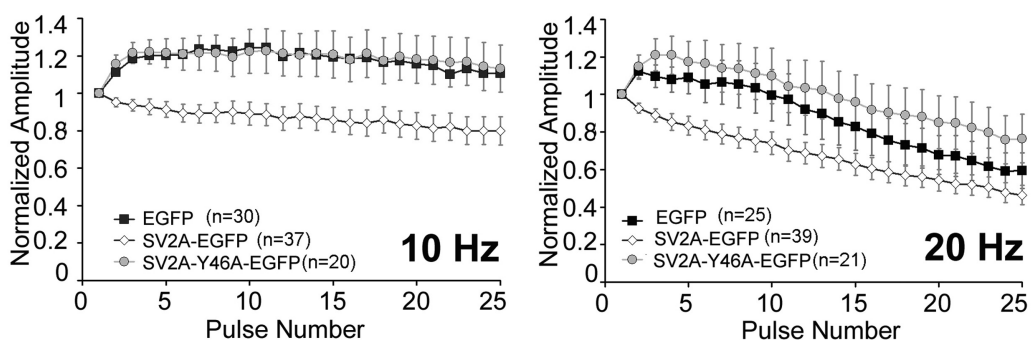

Figure 2. SV2A-EGFP but not SV2A-Y46A-EGFP restores normal neurotransmission to neurons from SV2A/B DK0 mice. Autaptic hippocampal neurons cultured from SV2A/B DK0 mice were infected with lentivirus encoding EGFP, SV2A-EGFP, or SV2A-Y46AEGFP and analyzed for features of the SV2 knock-out phenotype using whole-cell voltage-clamp recordings. Error bars represent SEM. $\boldsymbol{a}, \boldsymbol{b}$, SV2A but not SV2A-Y46A increases EPSC magnitude. Shown are average EPSC amplitudes ( $\boldsymbol{a}$ ) and total charge transfer (b) recorded from neurons expressing the indicated construct. The number of cells analyzed is indicated within the bars. The average EPSC amplitude and total charge transfer in neurons expressing SV2A were significantly larger than in neurons expressing either EGFP or SV2A-Y46A-EGFP as determined by Student's $t$ test (unpaired, two tailed). ${ }^{*} p<0.05$; ${ }^{* *} p<0.01$. For EPSC amplitudes: EGFP versus wild type (WT), $p=0.0008 ;$ Y 46 A versus EGFP, $p=0.329 ;$ Y 46 A versus WT, $p=0.016$. For total charge transfer: EGFP versus WT, $p=0.0006$; Y46A versus EGFP, $p=0.266$; Y46A versus WT, $p=0.017$. c, SV2A but not SV2A-Y46A restores paired-pulse depression. The EPSC amplitude in response to two stimuli separated by $45 \mathrm{msec}$ was expressed as the ratio of the second to the first response. Data were evaluated using Student's t test (unpaired, two-tailed). ${ }^{* *} p<0.01$. For EGFP versus WT, $p=0.0005$; for Y46A versus EGFP, $p=0.074$; for Y46A versus WT, $p=0.00003$. $d$, SV2A but not SV2A-Y46A restores synaptic depression in response to stimulus trains. Shown are averaged normalized responses to $10 \mathrm{~Hz}$ (left) or $20 \mathrm{~Hz}$ (right) stimulus trains. EPSC amplitudes were normalized to the first response in the train. The number of cells analyzed is indicated in the parentheses.

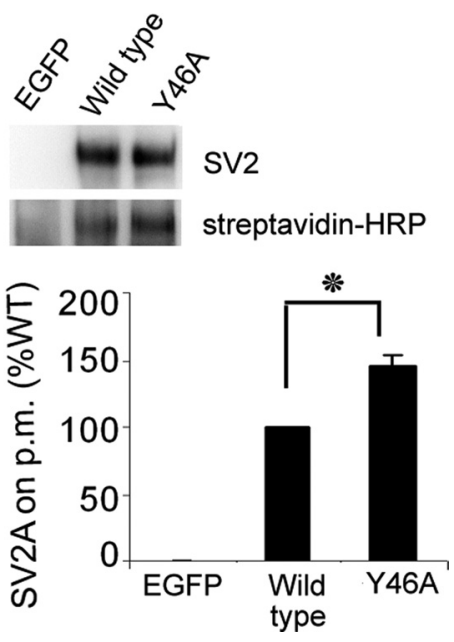

Figure 3. Reduced endocytosis of SV2A-Y46A. The proportion of SV2 on the plasma membrane (p.m.) was measured by surface biotinylation followed by immunoprecipitation with an anti-SV2A antibody. Immunoprecipitated material was analyzed by Western blot for both SV2 and biotin content. The top panel shows a representativeblotseries. The ratio of biotin to SV2 was calculated for each lane and normalized to the ratio obtained from neurons expressing wild-type SV2A in the same blot. Student's ttest was used for statistical analysis of the data. The error bars representSEM $(n=3) .{ }^{*} p<$ 0.05 . For SV2A immunoprecipitated samples, for Y46A versus wild type, $p=0.033$.

ice-cold homogenization buffer (0.32 м sucrose, 10 mM HEPES, pH 7.4, $1 \times$ protease inhibitor mixture; Roche) and centrifuged at $1000 \times g$ for 10 min at $4^{\circ} \mathrm{C}$. Triton $\mathrm{X}-100$ was added to the resulting supernatants to a final concentration of $1 \%$. The mixtures were extracted for $1 \mathrm{~h}$ at $4^{\circ} \mathrm{C}$.

Insoluble material was removed by centrifuging at $19,000 \times g$ for $30 \mathrm{~min}$. The protein concentration of the extract was determined by the BCA method using bovine serum albumin as a standard.

Recombinant SV2A-FLAG fusion proteins were expressed and purified as described previously (Yao and Bajjalieh, 2008). The concentration of purified fusion proteins was determined by running aliquots on SDS-PAGE gels followed by Coomassie blue staining with bovine serum albumin as standards. SV2A-FLAG fusion proteins attached to anti-FLAG M2 affinity beads (3-6 $\mu \mathrm{g}$ SV2A fusion protein) were incubated with $1 \mathrm{mg}$ of brain extract for at least $3-16 \mathrm{~h}$ at $4^{\circ} \mathrm{C}$ in the presence of $2 \mathrm{~mm}$ EGTA. A parallel reaction was set up with protein-free beads. After incubation beads were washed with extraction buffer four times, bound proteins were eluted with SDS-PAGE sample buffer or $3 \times$ FLAG peptide.

Proteomic analysis of SV2A binding proteins. SV2 N-terminus pull-down samples were eluted with $3 \times$ FLAG peptide, concentrated by trichloroacetic acid precipitation, and resuspended in SDS-PAGE sample buffer. Samples were electrophoresed briefly into the gel matrix, after which gel slices were cut out and washed in $50 \%$ methanol, $7.5 \%$ acetic acid followed by $5 \%$ methanol, $7.5 \%$ acetic acid for $1 \mathrm{~h}$ each. Gel slices were then washed three times in distilled water. Proteins in the gel slices were subjected to trypsin digestion, and the peptides were extracted and processed at the Proteomics Facility at the Fred Hutchinson Cancer Research Center (Seattle, WA). Tryptic peptides were analyzed using a Thermo Finnigan LTQFT. A mouse proteome database was searched using X! Tandem software. PeptideProphet was used to assign scores for each peptide, and scores of $\geq 0.65$ were used, which corresponded to a $\sim 5 \%$ error rate. Three biological replicates were analyzed at the same time.

Surface biotinylation. Conventional cultures of hippocampal neurons were rinsed two times with cold PBS, $\mathrm{pH}$ 8.0, and biotinylated by incubating with $0.5 \mathrm{mg} / \mathrm{ml}$ EZ-Link Sulfo-NHS-LC-LC-Biotin (Pierce) for 25 $\min$ at $4^{\circ} \mathrm{C}$. Cultures were washed with PBS, $\mathrm{pH} 7.4$, and incubated with $100 \mathrm{~mm}$ glycine in PBS for $15 \mathrm{~min}$ to quench the reaction. After two rinses with PBS, neurons were harvested in solubilization buffer $(150 \mathrm{~mm} \mathrm{NaCl}$, $50 \mathrm{~mm}$ Tris- $\mathrm{HCl}$, pH 7.4, 1 mm EDTA, $1 \%$ NP-40, $1 \times$ protease inhibitor mixture) and extracted for $30 \mathrm{~min}$ at $4^{\circ} \mathrm{C}$. Insoluble material was removed by centrifuging at $19,000 \times g$ for $20 \mathrm{~min}$. The supernatants were subjected to immunoprecipitation. Polyclonal anti-SV2A or antisynaptotagmin 1 antibodies were conjugated to protein A Sepharose beads (GE Healthcare) and incubated with the culture extract at $4^{\circ} \mathrm{C}$ for $\geq 2 \mathrm{~h}$. Beads were washed with solubilization buffer four times, after which the immunoprecipitated complexes were eluted with SDS-PAGE sample buffer. Total immunoprecipitated protein was determined by Western blot using monoclonal antibodies against SV2 or synaptotagmin 1. Antibody binding was detected with ECL reagent (Pierce). Biotinylated proteins were detected with streptavidin-HRP and visualized with ECL reagent. The net intensity of labeled bands was quantified using a Kodak Image Station 440, and only nonsaturated images were used for quantification analysis.

Immunoisolation of synaptic vesicles. Synaptic vesicles from wild-type and SV2A/B DKO mice were prepared by density centrifugation followed by immunoisolation. Brains were disrupted by blending in liquid nitrogen followed by homogenization in $0.3 \mathrm{~m}$ sucrose using a glass-Teflon homogenizer. Homogenates were centrifuged at $100,000 \times g$ for $1 \mathrm{~h}$, and the resulting supernatants were loaded onto a $0.6 / 1.5 \mathrm{~m}$ sucrose step 
Table 1. A screen for proteins that bind preferentially to the amino terminus of wild-type SV2A

\begin{tabular}{|c|c|c|c|c|c|c|}
\hline UniProtKB/Swiss-Prot entry name & Primary accession number & Gene name & Protein description & Total pep. hits (WT) & Total pep. hits (Y46A) & WT/Y46A \\
\hline \multicolumn{7}{|l|}{ Endocytosis related } \\
\hline AMPH_MOUSE & Q7TQF7 & Amph & Amphiphysin & 51 & 22 & 2.3182 \\
\hline BIN1_MOUSE & 008539 & Bin1 & Amphiphysin-like protein/amphiphysin 2 & 57 & 17 & 3.3529 \\
\hline Q8CC13_MOUSE & Q8CC13 & Ap1b1 & AP-1 complex subunit $\beta-1$ & 71 & 0 & $\mathrm{n} / \mathrm{a}$ \\
\hline AP2S1_MOUSE & P62743 & Ap2s1 & AP-2 complex subunit $\sigma$-1 & 16 & 0 & $\mathrm{n} / \mathrm{a}$ \\
\hline Q8C2J5_MOUSE & Q8C2J5 & Ap2a2 & AP-2 complex subuint $\alpha-2$ & 172 & 14 & 12.286 \\
\hline AP2M1_MOUSE & P84091 & Ap2m1 & AP-2 complex subunit $\mu-1$ & 143 & 13 & 11 \\
\hline AP2B1_MOUSE & Q9DBG3 & Ap2b1 & AP-2 complex subunit $\beta-1$ & 274 & 28 & 9.7857 \\
\hline AP2A1_M0USE & P17426 & Ap2a1 & AP-2 complex subunit $\alpha-1$ & 237 & 27 & 8.7778 \\
\hline AP3B2_MOUSE & Q9JME5 & $A p 3 b 2$ & AP-3 complex subunit $\beta-2$ & 17 & 0 & $\mathrm{n} / \mathrm{a}$ \\
\hline AAK1_MOUSE & Q3UHJO & Aak1 & AP2-associated protein kinase 1 & 79 & 2 & 39.5 \\
\hline Q80ZL3_MOUSE & Q80ZL3 & Eps15 & Epidermal growth factor receptor pathway substrate 15 & 26 & 0 & $\mathrm{n} / \mathrm{a}$ \\
\hline EP15R_MOUSE & Q60902 & Eps15/1 & Epidermal growth factor receptor substrate 15 -like 1 & 62 & 0 & $\mathrm{n} / \mathrm{a}$ \\
\hline ITSN1_MOUSE & Q9ZOR4 & Itsn1 & Intersectin 1 & 82 & 0 & $\mathrm{n} / \mathrm{a}$ \\
\hline NUMBL_MOUSE & 008919 & Numbl & Numb-like protein & 13 & 0 & $\mathrm{n} / \mathrm{a}$ \\
\hline SGIP1_MOUSE & Q8VD37 & Sgip1 & Endophilin interacting protein 1 & 38 & 0 & $\mathrm{n} / \mathrm{a}$ \\
\hline \multicolumn{7}{|l|}{ Other proteins } \\
\hline KCC2B_MOUSE & P28652 & Camk2b & CaM-kinase II $\beta$ chain & 13 & 5 & 2.6 \\
\hline Q58EU8_MOUSE & Q58EU8 & $\operatorname{lgk}-C$ & Igk protein & 34 & 10 & 3.4 \\
\hline P5CR3_MOUSE & Q9DCC4 & Pycrl & Pyrroline-5-carboxylate reductase 3 & 34 & 14 & 2.4286 \\
\hline Q545A2_MOUSE & Q545A2 & SIC25a5 & Solute carrier family 25 , member 5 & 11 & 5 & 2.2 \\
\hline PURA_MOUSE & P42669 & Pura & Transcriptional activator protein Pur- $\alpha$ & 16 & 6 & 2.6667 \\
\hline TBB2B_MOUSE & Q9CWF2 & $T u b b 2 b$ & Tubulin $\beta-2 \mathrm{~B}$ chain & 12 & 0 & $\mathrm{n} / \mathrm{a}$ \\
\hline
\end{tabular}

Listed is a subset of proteins that bound SV2A ${ }^{1-163}$-FLAG at higher levels than an equivalent protein carrying the Y46A mutation. WT, Wild-type; $n / a$, not applicable; pep., peptide.

gradient. Gradients were centrifuged at $250,000 \times g$ for $2.5 \mathrm{~h}$. Synaptic vesicles were collected at the interface of the 0.6 and $1.5 \mathrm{~m}$ sucrose steps. Vesicles were exchanged into PBS by passing them through an equilibrated gel filtration P10 column (Bio-Rad). Protein content was determined using the Bradford assay (Bio-Rad) with BSA as a standard. For immunoisolation, polyclonal anti-synaptophysin antibody was bound to protein A Sepharose, CL-4B beads and incubated overnight at $4^{\circ} \mathrm{C}$ with $500 \mu \mathrm{g}$ of precleared (with empty beads) synaptic vesicles. Beads were washed with PBS and processed for Western blot analysis.

Western blot analyses. The linear range was determined for each antibody used in Western blot analyses as reported previously (Morgans et al., 2009). An amount of mouse brain postnuclear supernatant that fell within the linear range (e.g., $4 \mu \mathrm{g}$ for anti-synaptotagmin) was assayed with actin serving as a loading control. In all cases, the linear range for anti-vesicle protein antibodies fell within the linear range of anti-actin.

Statistical analysis. The data shown in Figures 2, 3, 5-7, and 9 are presented as means \pm SEM. Statistical significance was determined using Student's $t$ test.

\section{Results}

In an effort to understand SV2's molecular action, we mutated residues predicted to underlie proposed actions of SV2 and assessed their ability to rescue normal neurotransmission in neurons from SV2A/B double knock-outs. SV2A contains two tyrosine-based endocytosis motifs, termed YXXФ motifs, where $\mathrm{Y}$ denotes tyrosine, $\mathrm{X}$ denotes any amino acid, and $\Phi$ denotes a hydrophobic amino acid. The first endocytosis motif is at amino acids 46-49 with sequence YSRF, which is in SV2A's cytoplasmic $\mathrm{N}$ terminus. The second motif is at the end of the cytoplasmic loop preceding transmembrane domain seven at amino acids 443-446 with sequence YRRI (Fig. 1a). The first motif is shared by SV2C, and a variant sequence (YRMN) is present at the second site in SV2B. Peptides containing the YXXФ motif from SV2A stimulate the binding of the clathrin adaptor AP2 to synaptotagmin (Haucke and De Camilli, 1999). Thus, these domains are predicted to facilitate vesicle protein endocytosis.

To investigate the functional role of these putative endocytosis motifs in SV2, we generated SV2A-EGFP fusion proteins carrying a tyrosine-to-alanine mutation in one or both of the motifs. The resulting proteins, SV2A-Y46A, SV2A-Y443A, and SV2A-Y46A/ Y443A, were expressed in hippocampal neurons cultured from SV2A/B DKO mice using a lentivirus expression system. Wildtype and mutant fusion proteins were all produced by neurons. However, coimmunolabeling with anti-SV2A and an antibody against the synaptic vesicle protein synaptophysin revealed that only wild-type SV2A and SV2A-Y46A were located at synapses (Fig. 1b). Thus, mutation of Y443 disrupts trafficking of SV2AEGFP. This mislocalized mutant was therefore not studied further. SV2A-Y46A, which did traffic to synapses, was expressed at comparable levels to wild-type SV2A as determined by Western blot analysis of conventional cultures (Fig. 1c). The correct localization of the SV2A-Y46A mutant permitted analysis of its function in neurons.

\section{Mutation of the first endocytosis motif in SV2A renders it nonfunctional and increases its surface expression}

The hallmark features of neurons lacking SV2 are reduced actionpotential-induced vesicle fusion and reduced synaptic depression (Custer et al., 2006; Chang and Sudhof, 2009). Both of these reflect reduced synaptic release probability. To evaluate the role of the endocytosis motif in SV2A function at the synapse, we compared the ability of wild-type SV2A and SV2A-Y46A to rescue EPSC amplitude and synaptic depression in autaptic hippocampal neurons from SV2A/B DKO mice. We assessed the effects of expressing EGFP, SV2A-EGFP, and SV2A-Y46A-EGFP on EPSC amplitude and total charge transfer, increased pairedpulse ratio (the ratio of a second response in relation to the first in a pair of stimuli), and decreased synaptic depression (Custer et al., 2006; Chang and Sudhof, 2009). Lentiviral-mediated expression of SV2A-EGFP significantly increased the average EPSC amplitude and total charge transfer compared to DKO neurons expressing EGFP (Fig. 2a,b). Likewise, normal paired-pulse ratios and synaptic depression were restored in neurons expressing SV2A-EGFP (Fig. $2 c, d$ ). Similar results have been reported for cultures of cortical neurons expressing EGFP-SV2A (Chang and 


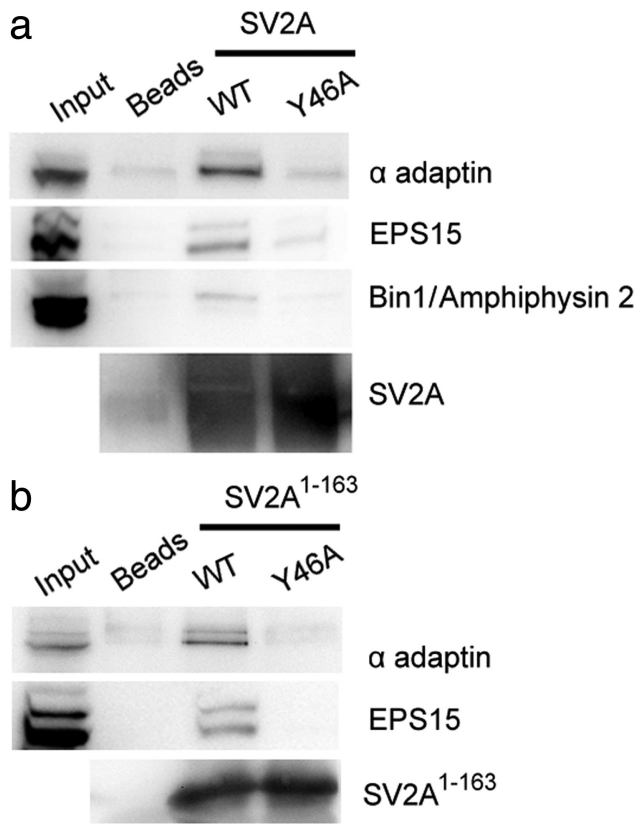

Figure 4. The Y46A mutation disrupts SV2A binding to endocytosis-related proteins. $\boldsymbol{a}$, Decreased binding of the adaptor proteins AP2 ( $\alpha$ adaptin), EPS15, and amphiphysin 2 (Bin 1) to SV2A-Y46A. SV2A-FLAG was generated in HEK293 fibroblasts and purified with anti-FLAG affinity resin. Beads were incubated with a Triton X-100 extract of mouse brain, after which bound proteins were identified by Western blot. When compared to wild-type SV2A, SV2A-Y46A demonstrated decreased binding to all three proteins. The blot shown is representative of three independent experiments. $\boldsymbol{b}$, Decreased binding of the adaptor proteins AP2 ( $\alpha$ adaptin) and EPS15 to the N terminus of SV2A-Y46A. A binding assay as in $a$ performed with SV2A ${ }^{1-163}$-FLAG fusion proteins is shown. In this case, the Y46A mutant demonstrated decreased binding to AP2 ( $\alpha$ adaptin) and EPS15. The blot shown is representative of six independent experiments.

Sudhof, 2009). Thus, the addition of EGFP to SV2A does not impair its function at the synapse. This finding also indicates that the neurotransmission deficit in neurons from SV2A/B DKO mice is attributable to the absence of SV2.

In contrast to wild-type SV2A-EGFP, expression of SV2AY46A-EGFP did not significantly increase EPSC amplitude or total charge transfer (Fig. $2 a, b$ ). Nor did it restore synaptic depression. Both paired-pulse ratios and responses to 10 and $20 \mathrm{~Hz}$ stimulus trains demonstrated facilitation, unlike the depression observed in neurons expressing wild-type SV2A (Fig. $2 c, d$ ). Because EPSC size and synaptic depression are indicative of synaptic release probability (Dobrunz and Stevens, 1997), these findings indicate that SV2A-Y46A, unlike wild-type SV2A, did not restore normal neurotransmission at synapses. This suggests that tyrosine 46 is essential to normal SV2 function.

To determine whether mutation of this endocytosis motif affects the endocytosis of SV2, we compared the proportion of SV2 on the plasma membrane by labeling surface proteins with biotin. For these studies, we used conventional cultures of hippocampal neurons from SV2A/B DKO mice because they contain many more neurons and fewer astrocytes than autaptic cultures. Cultures were infected with lentivirus encoding EGFP, SV2A-EGFP, or SV2A-Y46A-EGFP on DIV 1-3. At DIV 13-15, 90-95\% of neurons were expressing the proteins. Surface proteins were labeled using a membrane-impermeant reactive biotin reagent, after which cultures were harvested, extracted in NP-40, and subjected to immunoprecipitation using an antibody directed against SV2A. The precipitated protein was subjected to two Western analyses, one to probe for the total amount of SV2A precipitated and one for SV2A biotinylation to measure the
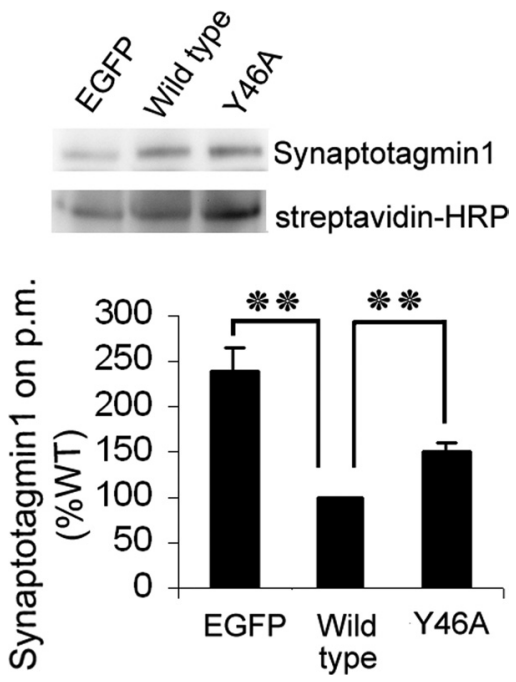

Figure 5. Reduced endocytosis of synaptotagmin 1 in neurons expressing SV2A-Y46A. The proportion of synaptotagmin on the plasma membrane was measured by surface biotinylation followed by immunoprecipitation with an anti-synaptotagmin 1 antibody. Immunoprecipitated material was analyzed by Western blot for both synaptotagmin and biotin content. The top panel shows a representative blot series. The ratio of biotin to synaptotagmin was calculated for each lane and normalized to the ratio obtained from neurons expressing wild-type (WT) SV2A in the same blot. Student's t test was used for statistical analysis of the data. The error bars represent SEM $(n=6) .{ }^{* *} p<0.01$. For EGFP versus WT, $p=0.004$; for Y46A versus WT, $p=0.003$. Expression of both SV2 proteins decreased biotinylation of synaptotagmin, but SV2A-Y46A did so to a lesser degree than wild-type SV2.
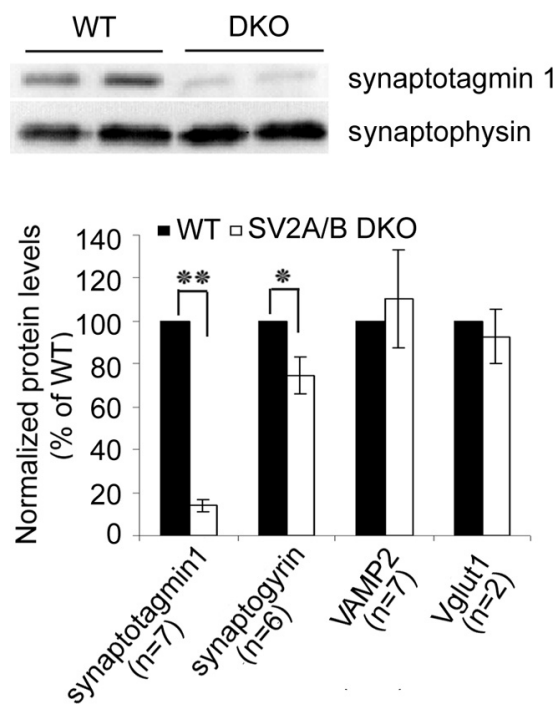

Figure 6. Synaptic vesicles from SV2A/B DKO mice have less synaptotagmin. Synaptic vesicles were prepared from wild-type (WT) or SV2A/B DK0 mice by density centrifugation followed with anti-synaptophysin immunoisolation. Immunoisolated synaptic vesicles were analyzed by Western blot for synaptotagmin 1, synaptogyrin, synaptobrevin/VAMP2, Vglut1, and synaptophysin. Top, A representative blot, containing duplicate samples of vesicles from WT and DKO mice, detected by anti-synaptotagmin 1 and synaptophysin antibodies. Bottom, Average protein to synaptophysin ratio for the indicated proteins. Data were analyzed by Student's $t$ test (unpaired, two-tailed). The number of times each study was replicated $(n)$ is indicated for each protein. The error bars represent SEM. ${ }^{*} p<0.05 ;{ }^{* *} p<0.01$.

amount that was on the neuronal surface. The intensity of the biotinylation signal was expressed as a ratio of the total SV2 signal. Values were normalized within each experiment to the ratio obtained from cultures expressing wild-type SV2A. Compared this way, we found that neurons expressing SV2A-Y46A 
a<smiles>O=C1C=CS(=O)(=O)C1</smiles>

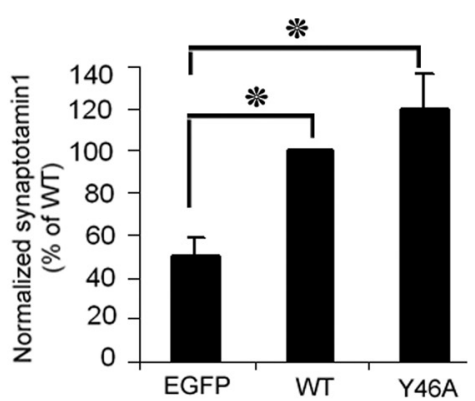

b

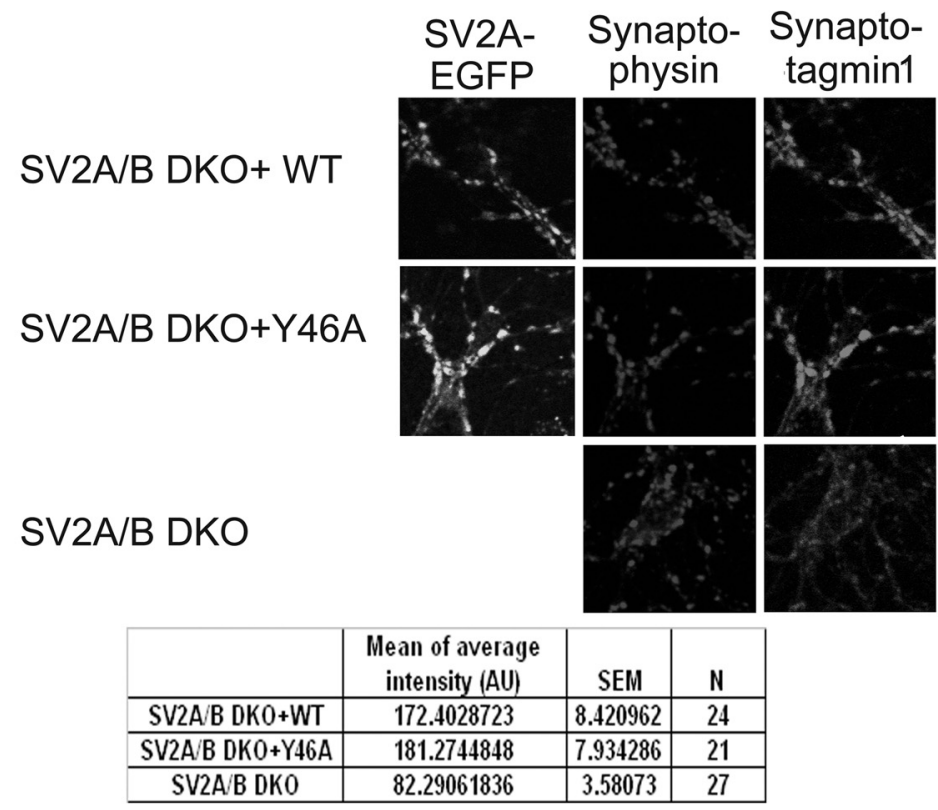

Synapto- Synapto-

C

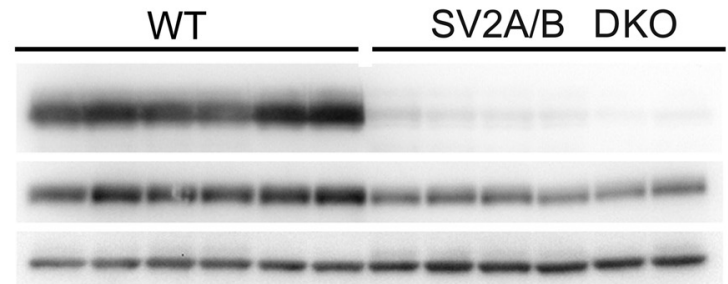

SV2

\section{Synaptotagmin 1}

actin

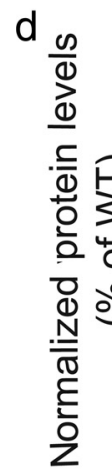

\section{-WT $\quad$-SV2A/B DKO}

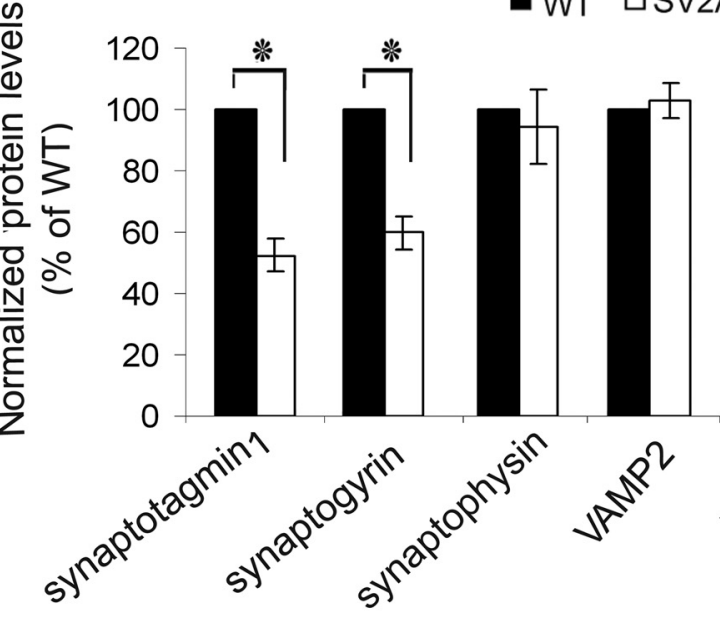

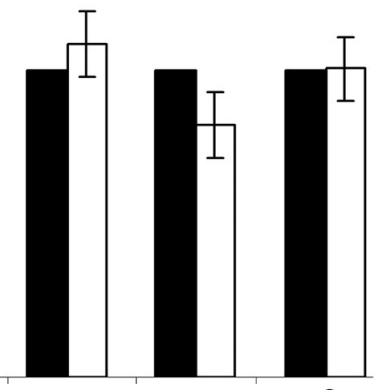

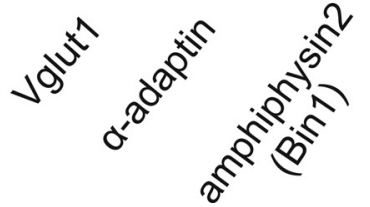

Figure 7. Expression of SV2 affects synaptotagmin expression. $\boldsymbol{a}$, Synaptotagmin expression is significantly reduced in neurons cultured from SV2A/B DK0 mice. Shown is a representative Western blot of cultured hippocampal neurons from SV2A/B DKO mice expressing the indicated SV2A protein. Cell extracts were probed with anti-synaptotagmin 1 and anti-actin, which served as a loading control. For each lane, the net intensity of synaptotagmin 1 to actin was expressed as a ratio, and the average ratio for wild-type (WT) SV2A was set to 100\%. Ratios for other lanes within the same blot were then normalized to that value. The graph

had an average of $\sim 45 \%$ more biotinylated SV2A (Fig. 3). Thus, the endocytosis motif at the $\mathrm{N}$ terminus of SV2A plays a role in its internalization.

\section{The endocytosis motif in SV2A mediates binding to proteins involved in endocytosis}

To begin to understand how the tyrosinebased motif of SV2A contributes to endocytosis, we performed a proteomic screen to identify protein interactions dependent on an intact endocytosis motif. We used recombinant proteins corresponding to amino acids 1-163 of SV2A or SV2AY46A as bait to screen mouse brain extract for proteins that preferentially bound wild-type SV2A. Associated proteins were subjected to trypsin digestion followed by reverse-phase liquid chromatography tandem mass spectrometry. Data analysis and statistical scoring of peptide identifications were done with PeptideProphet, a program developed by Keller et al. (2002). Table 1 lists proteins that were identified in three replicates as having at least twofold more peptide fragments associated with wild-type SV2A ${ }^{1-163}$. Included are multiple clathrin adaptor proteins (for re-

\section{$\leftarrow$}

on the right represents data from four experiments. ${ }^{*} p<0.05$ by Student's $t$ test. For EGFP versus WT, $p=0.01$; for Y46A versus EGFP, $p=0.02$; for Y46A versus WT, $p=0.348$. $\boldsymbol{b}$, Synaptotagmin expression is significantly reduced in the synapses of neurons cultured from SV2A/B DKO mice. To measure synaptic levels of synaptotagmin, we performed immunolabeling of cultured neurons using anti-synaptophysin to identify synapses. Representative images at the top demonstrate decreased synaptotagmin 1 levels in the synapses of neurons from SV2A/B DKO mice. Expression of both wild-type SV2A and SV2A-Y46A increased synaptotagmin 1 levels. The table lists the mean average synaptotagmin labeling intensity from two independent experiments. Data were analyzed by Student's $t$ test (unpaired, two-tailed). For DKO versus DKO + WT, $p<0.00001$; for DK0 versus DK0 + Y46A, $p<$ 0.00001 . $N$ indicates the total number of images analyzed for each condition. AU, Arbitrary unit. c, Synaptotagmin expression is significantly reduced in SV2A/B DKO mouse brain. Shown is a Western blot of postnuclear supernatants (PNSS) from the brains of six wild-type and six SV2A/B DKO mice that was probed for SV2, synaptotagmin 1, and actin. The ratio of anti-synaptotagmin 1 to anti-actin was calculated for each lane and graphed in $\boldsymbol{d}$. The residual anti-SV2 labeling in the DKO samples represents SV2C. $\boldsymbol{d}$, SV2 selectively affects the expression of synaptotagmin and synaptogyrin in brain. Brain PNSs from six wild-type and six SV2A/B DKO mice were probed for synaptotagmin 1, synaptogyrin, synaptophysin, VAMP2, and Vglut1. Brain PNSs from four wild-type and seven SV2A/B DKO mice were probed for AP2 ( $\alpha$ adaptin) and amphiphysin 2 (Bin1). Actin content was assessed as a loading control for each sample. The ratio of query protein to actin was calculated for each lane, averaged, and graphed. Data were analyzed by Student's $t$ test (unpaired, two-tailed). The error bars represent SEM. ${ }^{*} p<0.05$. 
view, see Maldonado-Baez and Wendland, 2006), including all four subunits of AP2, subunits of AP1 and AP3, two isoforms of EPS15, and Numb-like protein. In addition to adaptors, other endocytosis-related proteins were identified, including endophilin interacting protein 1, intersectin 1, AP2-associated protein kinase 1, and two isoforms of amphiphysin.

In addition to the proteins included in Table 1, which was restricted to proteins for which $>10$ peptides were identified, there were several proteins with $<10$ peptides identified that demonstrated preferential binding to wild-type SV2A. These included synaptotagmin 1 (four peptides identified in wild-type samples vs one with the mutant) and Numb protein (six peptides vs zero). This may reflect a bias in the procedure (e.g., fewer tryptic cleavage sites or poor extraction from the gel matrix). On the other hand, it is also consistent with the endocytosis motif of SV2 having the highest affinity for endocytosis-related proteins. Note that the absence of proteins acting later in endocytosis (e.g., dynamin, synaptojanin) suggests that the $\mathrm{N}$ terminus of SV2A mediates interactions relevant to early endocytotic events. The high level of apparent binding to AP2 is consistent with SV2 acting at the point of cargo recognition.

To verify the results of the proteomic screen, we performed Western blot analyses on proteins isolated with SV2A's N terminus and also with full-length SV2A. Both the $\mathrm{N}$ terminus and full-length wild-type SV2A bound the clathrin adaptor proteins AP2- $\alpha$ and EPS15. Full-length SV2A showed weak but detectable binding to amphiphysin 2 (Bin1). In both cases, binding was higher in wild-type SV2A than in SV2A-Y46A (Fig. 4).

\section{SV2 influences synaptotagmin 1 trafficking and expression}

SV2 is part of a large protein complex that includes the synaptic vesicle protein synaptotagmin 1 (Bennett et al., 1992; Schivell et al., 1996, 2005). SV2 is hypothesized to influence the endocytosis of synaptotagmin because peptides corresponding to the endocytosis domain in SV2A increase binding of synaptotagmin to the adaptor protein, AP2 (Haucke and De Camilli, 1999). It was possible that the Y46A mutation might affect the endocytosis of synaptotagmin. To test this, we assessed the effects of the Y46A mutation on surface levels of the synaptic vesicle protein synaptotagmin 1. We found that expression of SV2A-Y46A resulted in a $\sim 50 \%$ increase in the proportion of biotinylated synaptotagmin 1 compared to neurons expressing wild-type SV2A (Fig. 5). An even greater (twofold) increase in biotinylated synaptotagmin 1 was observed in DKO cultures expressing EGFP (which lack $\mathrm{SV} 2 \mathrm{~A} / \mathrm{B}$ ). A less extensive analysis of the vesicle protein synaptophysin revealed no change in surface expression in neurons expressing SV2A-Y46A (data not shown). This suggests that the effect is specific to synaptotagmin and is consistent with synaptophysin's absence from the SV2 protein complex (Schivell et al., 1996).

If SV2 is required for proper trafficking of synaptotagmin to synaptic vesicles, we would expect vesicles isolated from mice lacking SV2 to contain less synaptotagmin. We therefore compared the amount of synaptotagmin in vesicles immunoisolated from either wild-type or SV2A/B DKO mouse brain. Vesicles were purified using an antibody to synaptophysin, and the amount of synaptotagmin in them was determined. The ratio of synaptotagmin to synaptophysin was $85 \%$ lower in vesicles from SV2A/B DKO mice (Fig. 6). In contrast, there was no decrease in the levels of VAMP2/synaptobrevin or the vesicular glutamate transporter 1 (VGlut1). There was a small (25\%) decrease in synaptogyrin, which may result from decreased expression (see below). Thus, SV2 appears to play a specific role in trafficking a

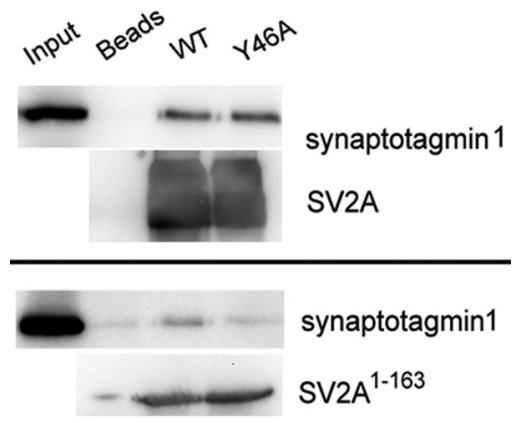

Figure 8. The Y46A mutation affects synaptotagmin binding to the $\mathrm{N}$ terminus of SV2A but not to full-length SV2A. Top, The Y46A mutation does not impact binding of synaptotagmin to full-length SV2A. In vitro binding assays were performed as in Figure 4 using full-length SV2AFLAG or SV2-Y46A-FLAG adsorbed to anti-FLAG resin. Resins were incubated with a Triton X-100 extract of mouse brain, after which associated proteins were identified by Western blot analysis. When compared to wild-type SV2A, SV2A-Y46A demonstrated similar binding to synaptotag$\min$. A representative blot from three independent experiments is shown. Bottom, $A n$ in vitro binding assay was performed with SV2A ${ }^{1-163}$-FLAG fusion proteins. In this case, the Y46A mutant demonstrated decreased binding to synaptotagmin. The blot shown is representative of six independent experiments.

subset of proteins to synaptic vesicles, with the largest effect on synaptotagmin. Coupled with our observation that SV2 influences the internalization of synaptotagmin, these results support the conclusion that SV2 plays a major role in regulating the amount of synaptotagmin in synaptic vesicles.

Immunoblot analyses of protein expression revealed that synaptotagmin levels were $\sim 50 \%$ lower in neurons lacking SV2. Expression of either wild-type SV2A or SV2A-Y46A restored total synaptotagmin expression (Fig. 7a). This decrease reflects diminished synaptic levels of synaptotagmin as determined by immunolabeling studies, which revealed a near identical (52\%) decrease in synapses lacking SV2A/B compared to synapses expressing either wild-type SV2A-EGFP or SV2A-Y46A-EGFP (Fig. $7 b$ ). The fact that both wild-type and mutant SV2A restored normal levels of synaptotagmin indicates that SV2A influences synaptotagmin expression via a mechanism that is independent of its affects on synaptotagmin trafficking, perhaps by inhibiting protein turnover.

The decreased synaptotagmin in neurons cultured from SV2A/B knock-out mice stands in contrast to previously published results that reported no change in brain levels of synaptotagmin in similar mice (Janz et al., 1999). We therefore repeated analyses of protein expression in wild-type and SV2A/B knockout mice, taking care to make sure the amount of protein assayed was in the linear range of the Western analysis. When performed in this way, we observed a selective decrease in synaptotagmin $(\sim 50 \%)$ (Fig. $7 c, d)$ and synaptogyrin $(\sim 40 \%)$ (Fig. $7 d$ ) in brain postnuclear supernatants from SV2A/B knock-out mice. Levels of other major vesicle proteins were not significantly altered, nor was there a difference in whole brain levels (Fig. $7 d$ ) or synaptic levels (data not shown) of the clathrin adaptor proteins AP2 and amphiphysin 2/Bin 1. Thus, SV2 exerts a selective effect on vesicle protein expression and is required for normal levels of synaptotagmin in vivo as well as in culture. The decreased expression of synaptogyrin matches the decrease in vesicle content, suggesting that vesicle content tracks expression levels for this protein. In contrast, the decrease in total synaptotagmin expression $(\sim 50 \%)$ is not as great as the decrease in synaptic vesicle content $(85 \%)$, consistent with SV2 influencing both the expression and trafficking of synaptotagmin. 
The tyrosine-based endocytosis motif in SV2 is required for binding to clathrin adaptor proteins

SV2 contains two synaptotagmin-binding sites: one, unique to SV2A and SV2C, that overlaps with the tyrosine-based endocytosis motif in the cytoplasmic $\mathrm{N}$ terminus, and a second, more diffuse, site that is present in all three SV2 isoforms (Schivell et al., 2005). To determine whether the Y46A mutation influences binding to synaptotagmin, we compared the ability of wild-type SV2A and SV2A-Y46A to bind synaptotagmin in vitro. Interestingly, we found that the Y46 mutation reduced synaptotagmin binding to the isolated $\mathrm{N}$ terminus but not to full-length SV2A (Fig. 8). Therefore it appears that SV2A's first endocytosis motif contributes to synaptotagmin binding, but that the second site (present in all isoforms) can support normal levels of synaptotagmin binding. Because full-length SV2A-Y46A demonstrated decrease binding to clathrin adaptors (Fig. 4), but not to synaptotagmin, it appears that SV2's interaction with clathrin adaptors does not occur through synaptotagmin. These findings support the interpretation that the N-terminal endocytosis motif of SV2A is a receptor for clathrin adaptor proteins.

Loss of SV2 has a minor effect on synaptic endocytosis

Given the effect of the Y46A mutation on clathrin adaptors, we sought to determine whether disrupted endocytosis of SV2 affects the retrieval of synaptic vesicles during synaptic activity. We assessed membrane internalization by measuring the uptake of the fixable FM dye, FM 4-64FX, in response to prolonged depolarization, and observed that synapses expressing SV2A-Y46A took up, on average, 15\% less FM 4-64FX dye than synapses expressing wild-type SV2A (Fig. 9). Because our analysis was limited to synaptic puncta that contained SV2A-EGFP, we did not quantify synaptic endocytosis in neurons from SV2A/B knock-out (DKO) mice expressing EGFP; however, qualitative analysis of the images indicated that a similar decrease was seen in neurons lacking SV2. This small difference could result from the decreased exocytosis that occurs in synapses lacking wild-type SV2, or it could indicate that SV2 provides a modest contribution to vesicle endocytosis. The magnitude of the effect, however, was too small to account for the changes in neurotransmission or vesicle content of synaptotagmin seen in neurons lacking SV2.

\section{Discussion}

Based on its structure, SV2 has been proposed to perform several actions, including transport of ions or small molecules into vesicles (Janz et al., 1999), providing the glycomatrix to the vesicle lumen (Reigada et al., 2003), and modulating the endocytosis of
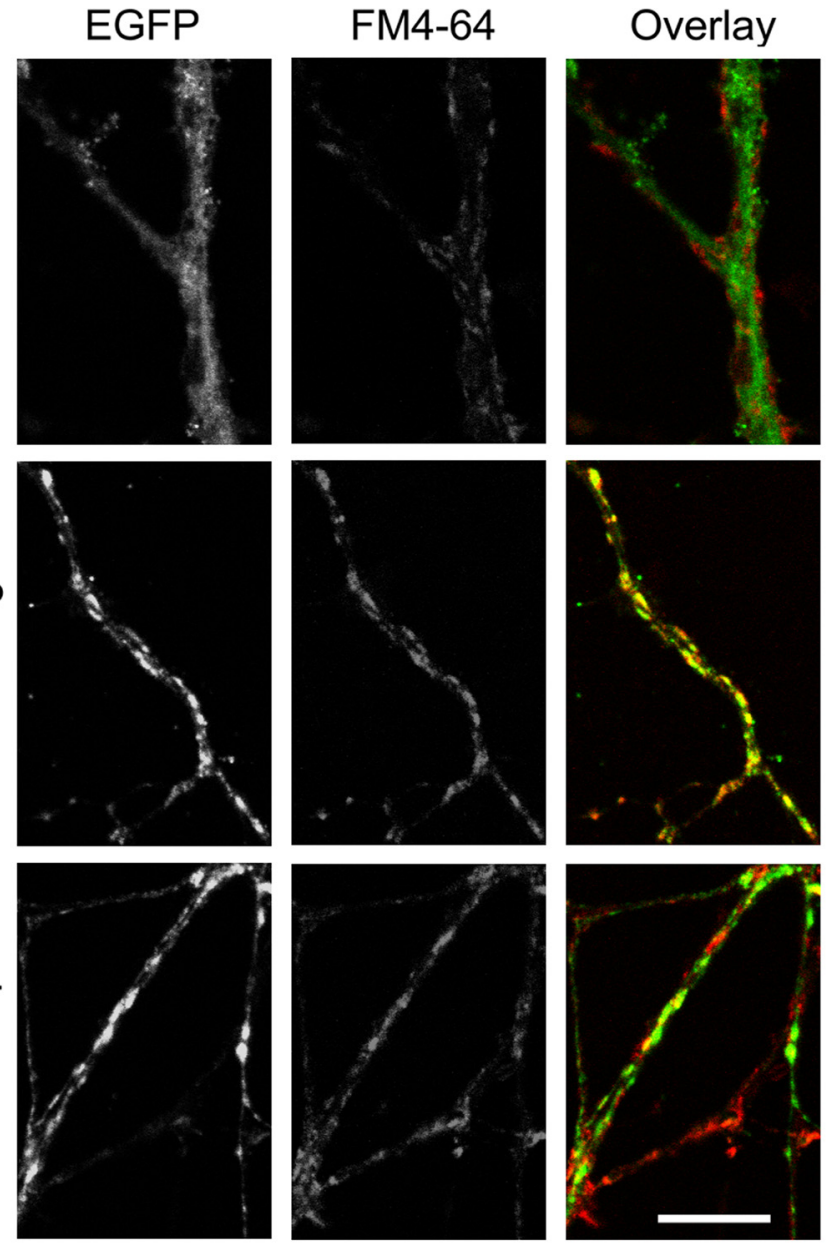

\begin{tabular}{|c|c|c|c|}
\hline & WT (mean \pm SEM) & Y46A (mean \pm SEM) & \\
& $\mathrm{N}=42$ & $\mathrm{~N}=38$ & $\boldsymbol{P}$ value \\
\hline Average intensity (AU) & $120.8 \pm 5.8$ & $100.1 \pm 4.7$ & 0.007 \\
\hline Normalized average intensity & $1.00 \pm 0.03$ & $0.84 \pm 0.05$ & 0.009 \\
\hline
\end{tabular}

Figure 9. Mutation of the first endocytosis motif in SV2A results in a minimal change in synaptic endocytosis. $\boldsymbol{a}$, Representative two-tailed). The data are from five experiments. N indicates the total number of images analyzed for each condition. AU, Arbitrary unit. There was a small decrease in FM 4-64 uptake in neurons expressing SV2A-Y46A.

vesicle proteins (Haucke and P. De Camilli, 1999). The findings presented here demonstrate that SV2 serves as a receptor for clathrin adaptor proteins and contributes to the proper trafficking of the vesicle protein synaptotagmin.

Mutation of Y46, which is part of a canonical YXX $\Phi$ adaptorbinding site (Owen and Evans, 1998) in the N terminus of SV2AEGFP, produced a protein that trafficked to synapses but did not support normal neurotransmission. In comparison to wild-type SV2A, SV2A-Y46A demonstrated reduced binding to multiple endocytosis-related proteins, including AP2, EPS15, and amphiphysin 2 (Bin1). Consistent with reduced binding to the mediators of endocytosis, the Y46A mutation resulted in lowered internalization of SV2. Because SV2A contains two YXXФ motifs, our results suggest that the N-terminal endocytosis motif in SV2A plays a dominant role in its endocytosis.

The findings presented here appear to conflict with the observation that an EGFP-SV2A fusion protein lacking amino acids 
1-107 of SV2A is functional (Chang and Sudhof, 2009). Although this could indicate that the Y46A mutation produces a dominantnegative effect, we note that EGFP protein contains a sequence that could serve as a tyrosine-based endocytosis motif (YGKL) at amino acids 40-43. Given the nearly identical position of this motif to that in the $\mathrm{N}$ terminus of native SV2A (amino acids 46-49), it is possible that the EGFP-SV2A fusion protein expressed in those studies was capable of binding clathrin adaptors.

Disruption of the endocytosis motif in SV2A's $\mathrm{N}$ terminus resulted in a higher proportion of synaptotagmin on the plasma membrane. This finding is consistent with SV2 playing an important role in the endocytosis of synaptotagmin. Although we cannot rule out the possibility that increased surface synaptotagmin was caused by increased delivery to the plasma membrane, when viewed in light of our finding that synaptic vesicles from SV2A/B knock-out mice contained significantly lower amounts of synaptotagmin, the most compatible conclusion is that SV2 regulates the endocytosis of synaptotagmin. Synaptotagmin has been proposed to be required for normal vesicle endocytosis (Jorgensen et al., 1995; Poskanzer et al., 2003; Nicholson-Tomishima and Ryan, 2004) and to be a clathrin adaptor receptor (Zhang et al., 1994; Chapman et al., 1998; Diril et al., 2006). Our findings suggest that SV2 contributes to any role synaptotagmin plays in endocytosis. We note with interest that our proteomic screen for proteins that bind the $\mathrm{N}$ terminus of SV2A did not identify stonin 2, a homolog of the $\mu$ subunit of AP2 (Martina et al., 2001) that mediates the endocytosis of synaptotagmin (Diril et al., 2006). SV2's ability to affect synaptotagmin endocytosis may therefore be through regulating synaptotagmin's ability to bind stonin 2 . Alternatively, by simultaneously engaging $\mu$ through its YXX $\Phi$ motif and interacting with synaptotagmin bound to stonin 2, SV2 may help concentrate clathrin adaptors in a way that insures the inclusion of synaptotagmin in vesicles.

In addition to influencing the trafficking of synaptotagmin, SV2 also exerts a selective effect on the expression of synaptotagmin. Synaptotagmin levels were considerably lower in both brain homogenates and neuron cultures from SV2A/B knock-out mice. A similar finding was reported in the retina of mice lacking SV2B (Lazzell et al., 2004; Morgans et al., 2009), which is the predominant SV2 isoform in photoreceptor synapses. This indicates that the ability to stabilize synaptotagmin is not mediated by the $\mathrm{N}$ terminus of SV2, since the A and B isoforms of SV2 have significantly different $\mathrm{N}$ termini. Consistent with this interpretation, both wild-type SV2A and SV2A-Y46A restored higher levels of synaptotagmin expression. The fact that expression of SV2AY46A rescued synaptotagmin expression but not its endocytosis suggests that the way in which SV2 affects synaptotagmin levels differs from the way it impacts synaptotagmin endocytosis.

Although the expression of SV2A-Y46A resulted in a modest decrease in endocytosis, the small magnitude of the effect, combined with normal vesicle number and morphology in SV2 knock-outs (Crowder et al., 1999; Janz et al., 1999), suggests that SV2 does not play a major role in vesicle biosynthesis. The smallness of the effect also suggests that reduced endocytosis is not likely to account for the significant decrease in neurotransmission observed in neurons expressing SV2A-Y46A. Finally, the disparity between the magnitude of SV2A-Y46A's effects on vesicle endocytosis and synaptotagmin internalization suggests that SV2 has a specific effect on synaptotagmin trafficking.

SV2 is essential for normal neurotransmission. In its absence, synaptic release probability decreases, a result that leads to a severe seizure phenotype and premature death (Crowder et al., 1999; Janz et al., 1999). The functional lesion in synapses lacking
SV2 occurs after vesicle docking (Custer et al., 2006) and before the formation of tightly associated SNARE (soluble $N$-ethylmaleimide-sensitive factor attachment protein receptor) complexes (Xu and Bajjalieh, 2001), indicating that SV2 contributes to the process that renders vesicles competent for calcium-stimulated fusion. Altered endocytosis could produce this phenotype by changing the protein composition of vesicles. Based on the findings presented here, a model emerges in which SV2A, via interaction with both synaptotagmin and clathrin adaptors, regulates the vesicle content of synaptotagmin. In the absence of SV2, synaptotagmin trafficking to synaptic vesicles becomes more random, leading to fewer vesicles with enough of the calcium sensor to trigger calcium-stimulated fusion. Thus, this action of SV2 can explain the neurotransmission deficit observed in SV2 knock-outs.

SV2's most striking feature is its structural similarity to major facilitator transporters (Pao et al., 1998), which has led to the widely held hypothesis that it transports something across the vesicle membrane. Although most proteins with transporter-like structure are transporters, there are several that perform other functions. A particularly interesting example is unc93b, a recently characterized transporter-like protein that plays a crucial role in trafficking Toll-like receptors to endosomes (Brinkmann et al., 2007; Kim et al., 2008). Our findings provide another example of a transporter-like protein acting as a modulator of protein trafficking to endosomes. Although we don't know whether the Y46A mutation also affects a transport activity of SV2, the finding that it renders SV2A nonfunctional and affects the endocytosis of synaptotagmin supports the conclusion that modulation of endocytosis is an essential action of SV2.

\section{References}

Bajjalieh SM, Franz G, Weimann JM, McConnell SK, Scheller RH (1994) Differential expression of synaptic vesicle protein 2 (SV2) isoforms. J Neurosci 14:5223-5235.

Bennett MK, Calakos N, Kreiner T, Scheller RH (1992) Synaptic vesicle membrane proteins interact to form a multimeric complex. J Cell Biol 116:761-775.

Brinkmann MM, Spooner E, Hoebe K, Beutler B, Ploegh HL, Kim YM (2007) The interaction between the ER membrane protein UNC93B and TLR3, 7, and 9 is crucial for TLR signaling. J Cell Biol 177:265-275.

Bushara KO, Malik T, Exconde RE (2005) The effect of levetiracetam on essential tremor. Neurology 64:1078-1080.

Chang WP, Sudhof TC (2009) SV2 renders primed synaptic vesicles competent for $\mathrm{Ca}^{2+}$-induced exocytosis. J Neurosci 29:883-897.

Chapman ER, Desai RC, Davis AF, Tornehl CK (1998) Delineation of the oligomerization, AP-2 binding, and synprint binding region of the $\mathrm{C} 2 \mathrm{~B}$ domain of synaptotagmin. J Biol Chem 273:32966-32972.

Crowder KM, Gunther JM, Jones TA, Hale BD, Zhang HZ, Peterson MR, Scheller RH, Chavkin C, Bajjalieh SM (1999) Abnormal neurotransmission in mice lacking synaptic vesicle protein 2A (SV2A). Proc Natl Acad Sci U S A 96:115268-115273.

Custer KL, Austin NS, Sullivan JM, Bajjalieh SM (2006) Synaptic vesicle protein 2 enhances release probability at quiescent synapses. J Neurosci 26:1303-1313.

De Smedt T, Raedt R, Vonck K, Boon P (2007) Levetiracetam: part II, the clinical profile of a novel anticonvulsant drug. CNS Drug Rev 13:57-78.

Diril MK, Wienisch M, Jung N, Klingauf J, Haucke V (2006) Stonin 2 is an AP-2-dependent endocytic sorting adaptor for synaptotagmin internalization and recycling. Dev Cell 10:233-244.

Dobrunz LE, Stevens CF (1997) Heterogeneity of release probability, facilitation, and depletion at central synapses. Neuron 18:995-1008.

Dunteman ED (2005) Levetiracetam as an adjunctive analgesic in neoplastic plexopathies: case series and commentary. J Pain Palliat Care Pharmacother 19:35-43.

Enggaard TP, Klitgaard NA, Sindrup SH (2006) Specific effect of levetiracetam in experimental human pain models. Eur J Pain 10:193-198. 
Haucke V, De Camilli P (1999) AP-2 recruitment to synaptotamin stimulated by tyrosine-based endocytic motifs. Science 285:1268-1271.

Janz R, Goda Y, Geppert M, Missler M, Sudhof TC (1999) SV2A and SV2B function as redundant $\mathrm{Ca}^{2+}$ regulators in neurotransmitter release. Neuron 24:1003-1016.

Jorgensen EM, Hartwieg E, Schuske K, Nonet ML, Jin Y, Jorvitz HR (1995) Defective recycling of synaptic vesicles in synaptotagmin mutants of Caenorhabditis elegans. Nature 378:196-199.

Keller A, Nesvizhskii AI, Kolker E, Aebersold R (2002) Empirical statistical model to estimate the accuracy of peptide identifications made by MS/MS and database search. Anal Chem 74:5383-5392.

Kim YM, Brinkmann MM, Paquet ME, Ploegh HL (2008) UNC93B1 delivers nucleotide-sensing toll-like receptors to endolysosomes. Nature 452: 234-238.

Kinrys G, Wygant LE, Pardo TB, Melo M (2006) Levetiracetam for treatment-refractory posttraumatic stress disorder. J Clin Psychiatry 67: 211-214.

Kinrys G, Worthington JJ, Wygant L, Nery F, Reese H, Pollack MH (2007) Levetiracetam as adjunctive therapy for refractory anxiety disorders. J Clin Psychiatry 68:1010-1013.

Lazzell DR, Belizaire R, Thakur P, Sherry DM, Janz R (2004) SV2B regulates synaptotagmin 1 by direct interaction. J Biol Chem 279:52124-52131.

Lynch BA, Lambeng N, Nocka K, Kensel-Hammes P, Bajjalieh SM, Matagne A, Fuks B (2004) The synaptic vesicle protein SV2A is the binding site for the antiepileptic drug levetiracetam. Proc Natl Acad Sci USA 101:9861-9866.

Maldonado-Baez L, Wendland B (2006) Endocytic adaptors: recruiters, coordinators and regulators. Trends Cell Biol 16:505-513.

Martina JA, Bonangelino CJ, Aguilar RC, Bonifacino JS (2001) Stonin 2: an adaptor-like protein that interacts with components of the endocytic machinery. J Cell Biol 153:1111-1120.

McGavin CL, John V, Musser WS (2003) Levetiracetam as a treatment for tardive dyskinesia: a case report. Neurology 61:419.

Morgans CW, Kensel-Hammes P, Hurley JB, Burton K, Idzerda R, McKnight GS, Bajjalieh SM (2009) Loss of the synaptic vesicle protein SV2B results in reduced neurotransmission and altered synaptic vesicle protein expression in the retina. PLoS ONE 4:e5230.

Nicholson-Tomishima K, Ryan TA (2004) Kinetic efficiency of endocytosis at mammalian CNS synapses requires synaptotagmin I. Proc Natl Acad Sci U S A 101:16648-16652.

Owen DJ, Evans PR (1998) A structural explanation for the recognition of tyrosine-based endocytotic signals. Science 282:1327-1332.

Pao SS, Paulsen IT, Saier MH Jr (1998) Major facilitator superfamily. Microbiol Mol Biol Rev 62:1-34.

Poskanzer KE, Marek KW, Sweeney ST, Davis GW (2003) Synaptotagmin I is necessary for compensatory synaptic vesicle endocytosis in vivo. Nature 426:559-563.

Price MJ (2004) Levetiracetam in the treatment of neuropathic pain: three case studies. Clin J Pain 20:33-36.

Reigada D, Diez-Perez I, Gorostiza P, Verdaguer A, Gomez de Aranda I, Pineda O, Vilarrasa J, Marsal J, Blasi J, Aleu J, Solsona C (2003) Control of neurotransmitter release by an internal gel matrix in synaptic vesicles. Proc Natl Acad Sci U S A 100:3485-3490.

Schivell AE, Batchelor RH, Bajjalieh SM (1996) Isoform-specific, calciumregulated interaction of the synaptic vesicle proteins SV2 and synaptotagmin. J Biol Chem 271:27770-27775.

Schivell AE, Mochida S, Kensel-Hammes P, Custer KL, Bajjalieh SM (2005) SV2A and SV2C contain a unique synaptotagmin-binding site. Mol Cell Neurosci 29:56-64.

Striano P, Elefante A, Coppola A, Tortora F, Zara F, Minetti C, Striano S (2007) Dramatic response to levetiracetam in post-ischaemic Holmes' tremor. J Neurol Neurosurg Psychiatry 78:438-439.

Woods SW, Saksa JR, Baker CB, Cohen SJ, Tek C (2008) Effects of levetiracetam on tardive dyskinesia: a randomized, double-blind, placebo-controlled study. J Clin Psychiatry 69:546-554.

$\mathrm{Xu}$ T, Bajjalieh SM (2001) SV2 modulates the size of the readily releasable pool of secretory vesicles. Nature Cell Bio 3:691-698.

Yao J, Bajjalieh SM (2008) Synaptic vesicle protein 2 binds adenine nucleotides. J Biol Chem 283:20628-20634.

Zhang JZ, Davletov BA, Sudhof TC, Anderson RGW (1994) Synaptotagmin $\mathrm{I}$ is a high affinity receptor for clathrin AP-2: Implications for membrane recycling. Cell 78:751-760.

Zhang W, Connor KM, Davidson JR (2005) Levetiracetam in social phobia: a placebo controlled pilot study. J Psychopharmacol 19:551-553.

Zivkovic SA, Costa G, Bond G, Abu-Elmagd KM (2008) Treatment of tardive dyskinesia with levetiracetam in a transplant patient. Acta Neurol Scand 117:351-353. 